Marquette University

e-Publications@Marquette

Speech Pathology and Audiology Faculty Research

and Publications

Speech Pathology and Audiology, Department of

$7-1-2011$

Speaking Rate Effects on Normal Aspects of Articulation: Outcomes and Issues

Jeffrey J. Berry

Marquette University, jeffrey.berry@marquette.edu

Published version. Perspectives on Speech Science and Orofacial Disorders, Vol. 21, No. 1 (July 2011): 15-26. DOI. (C) 2011 American Speech-Language-Hearing Association. Used with permission. 
Unless otherwise noted, the publisher, which is the American Speech-LanguageHearing Association (ASHA), holds the copyright on all materials published in Perspectives on Speech Science and Orofacial Disorders, both as a compilation and as individual articles. Please see Rights and Permissions for terms and conditions of use of Perspectives content: $\underline{\text { http://journals.asha.org/perspectives/terms.dtl }}$

\section{Speaking Rate Effects on Normal Aspects of Articulation: Outcomes and Issues}

Jeff Berry

Department of Speech Pathology and Audiology, Marquette University

Milwaukee, WI

\section{Abstract}

The articulatory effects of speaking rate have been a point of focus for a substantial literature in speech science. The normal aspects of speaking rate variation have influenced theories and models of speech production and perception in the literature pertaining to both normal and disordered speech. While the body of literature pertaining to the articulatory effects of speaking rate change is reasonably large, few speakergeneral outcomes have emerged. The purpose of this paper is to review outcomes of the existing literature and address problems related to the study of speaking rate that may be germane to the recurring theme that speaking rate effects are largely idiosyncratic.

\section{Introduction}

Modifications of speaking rate have long been assumed to reveal information about the mechanisms underlying articulatory control (Stetson, 1951). Many studies regarding normal aspects of speech motor control have embraced this assumption, evoking rate variation from speakers as a primary experimental manipulation. The observations reported in such studies and the speculations that have arisen from them have influenced theories and models of speech production and speech perception (e.g., Guenther, 1995; Lindblom, 1963; Lindblom \& Studdert-Kennedy, 1967; Saltzman \& Munhall, 1989).

Individuals appear to prefer speaking at a rate that is idiosyncratic and, yet, bounded by certain "acceptable" limits (Tsao $\&$ Weismer, 1997). Speaking rates outside these limits may be characteristic of certain motor speech disorders (Nishio \& Niimi, 2001, 2006; Weismer, Tjaden, \& Kent, 1995), and rate manipulations have seen wide clinical use, particularly with regard to dysarthria (Blanchet \& Snyder, 2010; Yorkston, Hakel, Beukelman, \& Fager, 2007) and stuttering (Blomgren \& Goberman, 2008; Logan, Roberts, Pretto, \& Morey, 2002;). Differentiating the impact of rate change from the impact of an external timing cue (such as a metronome) is a particularly poignant issue in the understanding of fluency enhancement strategies (Davidow, Bothe, Richardson, \& Andreatta, 2010). Thus, speaking rate is an important variable in theory, research, and practice regarding speech disorders.

Despite the relatively large quantity of speech science research pertaining to normal aspects of the articulatory effects of speaking rate, few speaker-general rules have emerged. In fact, the principal finding of research related to normal aspects of speaking rate is idiosyncrasy. The purpose of this paper is to review the literature pertaining to the articulatory effects of speaking rate change and present a discussion of methodological concerns that may be germane to the apparent elusiveness of a speaker-general model. 


\section{Kinematics}

Studies of the kinematic effects of speaking rate derive data using a variety of instrumental techniques (cf., Stone, 1997). The bulk of these studies have analyzed rate-related changes for a relatively small number of kinematic dimensions. These dimensions include the speed/velocity of selected articulatory movements, the distance over which the articulator moves during one or more speech gestures, and relative timing (phasing) of articulatory movements. At first glance, it would seem that varying speaking rate would have to involve altering at least one of these dimensions of movement. For example, to increase speaking rate, a speaker could

1. Move articulators an equivalent distance, but increase speed/velocity,

2. Maintain similar speed/velocity but reduce the size of movements so less distance has to be covered, or

3. Create greater overlap in articulatory movements associated with successive phonetic elements (increasing coarticulation/coproduction).

To date, the data suggest that the particular constellation of rate-induced changes in these parameters often differs between speakers, contexts, and articulators. These findings prompted Gay (1981) to argue that these rate-induced kinematic transformations are not only multidimensional, but also nonlinear, presumably suggesting that the relationships between a quantitative measure of rate, such as segment duration, and the various kinematic parameters do not change monotonically.

\section{Rate-Induced Variation in Articulatory Speed and Distance}

The term "speed" refers to the scalar magnitude of the velocity vector calculated for each position sample of a movement (Tasko \& Westbury, 2002). Since velocity is a vector quantity, it characterizes rate of change in a specified direction. Throughout this paper the terms "speed" and "velocity" will be used interchangeably, because both are common in the literature and they are conceptually related. In strict usage, however, these are different measures. Velocity is a characterization of the rate of change of a movement history along a single movement dimension (i.e., "up-down" or "front-back" or "side-to-side"). Alternatively, speed refers to rate of change in position without restriction to a single movement dimension. Consequently, speed is a characterization of the total rate of change of a movement trajectory. It is necessary to draw these distinctions because measurement of velocity implies that the local coordinate system defining a single movement dimension adequately captures the speech movements of interest and that this axis remains valid within and across speaker and context. For example, simply measuring the velocity of "up-down" movements of the tongue during vowel production is very likely to miss key aspects of vowel articulation. Measurement of speed avoids such an implication, making it immune to the influence of variation in the orientation of the local coordinate system within or across speaker and context.

Several studies of speaking rate effects on articulator movement velocities have reported increased "peak" velocities (velocity maxima) with increased speaking rate (Abbs, 1973; Adams, Weismer, \& Kent, 1993; Flege, 1988; Gay \& Hirose, 1973; Hertrich \& Ackermann, 2000; Kuehn $\&$ Moll, 1976; Ostry \& Munhall, 1985; Shaiman, 2001, 2002). This is consistent with the notion that, in order to speak faster, one must move one's articulators faster. However, these results have not always been consistent across articulators. For example, Hertrich and Ackermann (2000) collected tongue, lips, and jaw data from 9 speakers using electromagnetic articulography. Their speakers produced two speaking rates by speaking in sync with synthetic syllable stimuli presented binaurally. The authors concluded that there may be a distinction between rate effects on jaw versus lips and tongue, reporting increases in (amplitude scaled) peak velocity for jaw movements, but not the lips and tongue. McClean (2000) also reported data suggesting that the jaw and lips may differ with respect to the impact of rate change. His kinematic study of 9 speakers producing three speaking rates revealed both relative increases 
and decreases in articulator movement velocity across subjects during fast speech, with consistently reduced velocities during slow speech. Tasko and McClean (2004) found a similar pattern of complex velocity changes for 15 speakers. McClean (2000) concluded that subjects displayed different strategies to change rate and individual differences may be particularly apparent with regard to jaw movement. Other reports have indicated little or no evidence of changes in articulator velocities as a function of speaking rate (Bengueral \& Cohen, 1974; Kent $\&$ Moll, 1972) and individual differences with regard to the occurrence of velocity changes (Flege, 1988; Kuehn \& Moll, 1976; Ostry \& Munhall, 1985).

Studies that emphasize individual differences in rate effects on articulator velocity often included tongue movement data. Ostry and Munhall (1985) report that 1 of their 3 speakers increased peak tongue velocity to increase speaking rate. Flege (1988) reported equivalent findings with 1 of his 3 speakers. Conversely, Kent and Moll's (1972) study of 2 speakers revealed little evidence of a direct relationship between velocity and speaking rate. The authors observed instances of relatively increased tongue velocity at the slower of the 2 speaking rates coupled with proportional changes in movement distance.

Kuehn and Moll (1976) analyzed cineradiographic data from speakers producing three speaking rates. The authors reported that two of the speaking rates were kinematically indistinct ("normal" and "slow"), but that speakers produced distinctions between these rates and a "fast" speaking rate using different (speaker-specific) proportions of velocity and movement distance change. Speakers demonstrated patterns of velocity increase, decrease, or no change in articulator velocity with an increase in speaking rate.

Goozee, Lapointe and Murdoch (2003) studied tongue movements of 8 male talkers producing two rates (habitual and fast) during repetitions of / ta/ and / ka/. Four subjects exhibited no consistent changes in velocity, while 2 decreased velocity and 2 increased velocity to achieve faster rates. Seven of the 8 subjects reduced movement distances at increased rate. The authors suggested four different strategies to increase speaking rate (in order of apparent popularity): (a) reduce distance, (b) reduce velocity and distance, (c) increase velocity and decrease distance, and (d) increase velocity with no change in distance. These conclusions highlight the apparent idiosyncrasy within a single kinematic parameter (velocity) and underscore the possibility of interactions between multiple kinematic parameters. In a comparative study of 8 young and 8 older females, Goozee, Stephenson, Murcdoch, Darnell, and Lapointe (2005) found similar variability across subjects, albeit with an apparently pervasive reduction in movement distances with rate increase (across contexts and speaker groups). The authors suggested that such a pervasive articulatory undershoot effect may reflect a "reactive" (rather than "active") mechanism of rate control geared toward an economy of physical effort (Nelson, 1983). This interpretation was based primarily on the observation that the only changes in movement velocity (across rates) that were observed maintained a positive correlation with movement distance changes.

Several studies of speaking rate have reported evidence of a direct relationship between movement speed and distance (Abbs, 1973; Hertrich \& Ackermann, 2000; Kent \& Moll, 1972; Kuehn \& Moll, 1976; Ostry \& Munhall, 1985; Shaiman, 2001). It has been suggested that the ratio of these two variables changes systematically with movement duration (Kelso, VatikiotisBateson, Saltzman, \& Kay, 1985; Ostry \& Munhall, 1985) and that this value may be useful for describing articulator "stiffness" in a simple mass-spring model of articulation. The implication of such a model is that the ratio of (peak) speed/distance is a parameter that is explicitly modulated by the speech motor control system. This concept is potentially parsimonious, given that modulations of two variables can be accounted for by a single concept: "isochrony," the tendency to scale movement speed and distance to limit durational variation (Kelso et al., 1985; Viviani \& Cenzato, 1985).

The generalizability of such a conceptual model may seem questionable, particularly with regard to speaking rate change, considering that a direct relationship between the two variables is not ubiquitous (Abbs, 1973; Benguerel \& Cowan, 1974; Gay, 1981; Flege, 1988; 
Kent \& Moll, 1972; Kuehn \& Moll, 1976). In fact, while observations of increases in articulator speed with increases in speaking rate tend to be common (though, not "universal"), corresponding decreases in articulator movement distance tend to be more common than increases (Byrd \& Tan, 1996; Flege, 1988; Gay, Ushijima, Hirose, \& Cooper, 1974; Kent \& Moll, 1972; Ostry \& Munhall, 1985; Shaiman, 2001). Thus, across speaking rates, we might expect an inverse relationship between speed and distance to be more likely than a direct relationship. In fact, McClean and Tasko (2003) studied 3 speakers' lower lip and jaw movements across six rate conditions; defined using time-warped speaker-specific utterances as performance targets. All targets were based on proportional changes from the talker's habitual rate. Kinematic results suggested that the relationship between movement speed and distance appeared to be affected by rate.

Perkell, Zandipour, Matthies, and Lane (2002) studied 7 speakers producing CVCs in a carrier phrase at three speaking rates. Attempting to assess the implications of an "economy of effort" model (Nelson, 1983), these authors completed a performance space analysis (speed $\mathrm{x}$ distance $\mathrm{x}$ duration) and concluded that slow speech can be differentiated from habitual and fast speech due to the loss of the proportional relationship between movement duration and distance. The authors concluded that slow speech may be "un-natural" for speakers. The notion that slow speech is categorically different from habitual and fast speech is also reflected through the analysis of changes in velocity over time (so-called velocity profiles).

\section{Rate-Induced Variation in Velocity Profiles}

As an alternative to descriptions of the effect of rate of change on some discrete kinematic parameter (e.g., average or peak velocity), some researchers have suggested the analysis of more complete histories of articulator velocity (Adams et al., 1993; Munhall, Ostry, \& Parush, 1985; Ostry et al., 1987; Shaiman, Adams, \& Kimelman, 1997). These velocity profiles have been attributed great significance in some control models of human movement (Plamondon, 1995). The velocity profile provides a more complete view of the manner in which an articulatory movement is completed. For example, the velocity profile could be a relatively simple unimodal pattern where the articulator accelerates away from a starting position and then decelerates as it reaches the final position, much like a car moving from one stop light to the next with no traffic. Alternatively, the velocity profile might be more complex with multiple accelerations and decelerations, more akin to a car that is speeding up and slowing down to meet traffic demands.

Adams, Weismer, and Kent (1993) provide a detailed analysis of characteristics of velocity profiles for closing and opening movements of the tongue tip and lower lip conditioned by speaking rate changes. They analyzed kinematic data acquired from 5 speakers producing five speaking rates using a magnitude production task. The authors reported a tendency across speakers for single-peaked (unimodal), symmetrical profiles to be associated with faster speaking rates, and multi-peaked, asymmetrical profiles to be associated with slower speaker rates. They concluded that these observations may evidence changes in motor control strategies across the speaking rate continuum, with faster rates associated with preprogrammed, unitary movements, and slower rates associated with feedback driven, multiple sub-movements.

Figure 1, adapted from Berry (2003,) demonstrates comparable findings for dorsal tongue marker movements during the vowel in the word "bag" produced in a carrier phrase across a wide range of speaking rates. Note the apparent tendency for the speed histories (velocity profiles) to become less clearly unimodal as speaking rate slows (vowel duration increases). There is also an apparent tendency for the maximum speed to reduce and for the minimum speed to increase as vowel duration increases. These observations highlight the possibility that some pertinent effects of rate may not be clearly conveyed through single, discrete-time measures. 
Figure 1. Speed histories for a dorsal tongue marker during rate-varied productions of "bag" (embedded in a carrier phrase) by single speaker. Note the apparent tendency for the speed histories to become increasingly multi-modal as speaking rate slows (vowel duration increases).

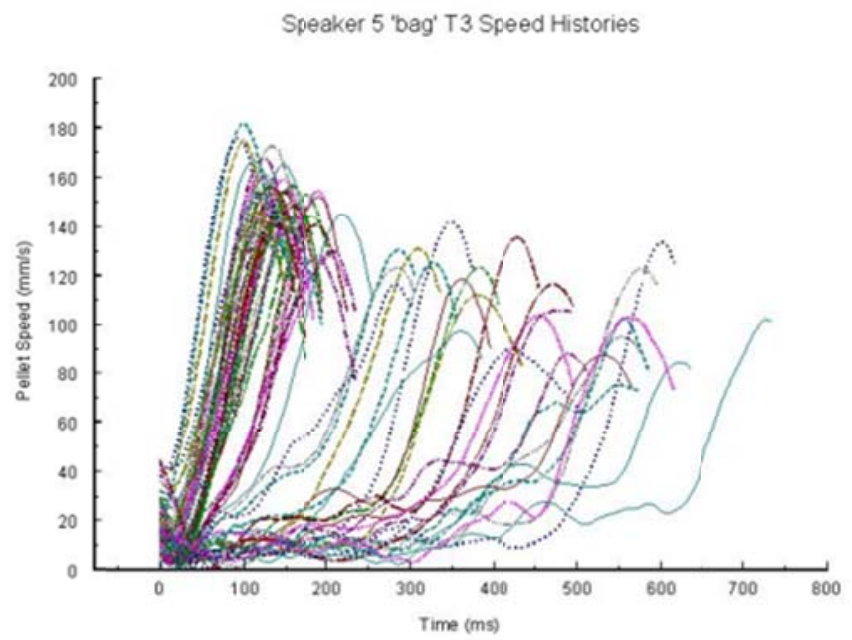

\section{Rate-Induced Variation in Phasing of Articulatory Movements}

Hypothetically, speaking rate can also be varied by varying the relative timing of successive articulatory movements, so that the overall duration of the event series is shortened. Increasing the "overlap" between two successive movements would decrease the overall duration and thus increase rate. We commonly refer to this process as coarticulation or coproduction. For example, when producing the first syllable in the word "object," the speaker will have to lower the mandible for the low back vowel and also begin to approximate the lips to produce the bilabial plosive that follows. When the speaker simply begins the lip approximation earlier (relative to the jaw lowering), the overall duration of the sequence can be shortened, thus increasing speaking rate. Measures that reflect inter-articulator temporal overlap or phasing have been studied. Like the other kinematic parameters, rate-induced effects on phasing appear to be far from predictable. The literature includes reports that increasing speaking rate results in (a) increased overlap, (b) no change in overlap, or (c) decreased overlap (Abbs, 1973; Boyce, Krakow, Bell-Berti, \& Gelfer, 1990; Byrd \& Tan, 1996; Engstrand, 1988; Shaiman, Adams, \& Kimelman, 1995; Shaiman, 2001, 2002).

Following Shaiman (2002), several different measures may reflect upon inter-articulator temporal overlap. Candidate measures include proporbe peak velocity, and the phase angle (defined using a normalization of movement displacement and velocity expressed in a polar coordinate a space). The procedure for deriving this latter parameter is detailed by Kelso et al. (1985). Shaiman (2002) reported that phase angle decreased (an upcoming movement occurred relatively sooner) with increasing rate when data were averaged across subjects. Shaiman et al. (1995) studied 8 speakers producing three speaking rates using a magnitude production task. Five speakers showed significant changes in phase angle across changes in speaking rate. Of these 5, 3 had decreased phase angles with shorter jaw cycle durations and 2 had increased phase angles with shorter jaw cycle duration. As is typical of results reported for rate effects on other kinematic measures, individual differences appear to the rule, rather than the exception.

Theoretical treatments that attribute great importance to phase rely on the assumption that inter-articulatory phase varies in some systematic way (Saltzman \& Byrd, 2000). Despite the lack of consistent speech production data supporting such an assumption, the plausibility of a phase-based kinematic model of speaking rate can be assessed in terms of the acoustic consequences of phase changes in an articulatory synthesizer. Figure 2, adapted from Berry 
(2002), shows a series of second formant (F2) patterns for the diphthong in the word "boy" generated with an articulatory synthesizer. The basic patterns of movement for the articulators of the synthesizer were derived from kinematic patterns measured for a single speaker saying the word "boy." By systematically shifting the temporal overlap of movements of the tongue with respect to lip and jaw, a series of rate-varied repetitions of the diphthong segment were generated. The top of Figure 2 displays the formant patterns resulting simply from shifting the degree of temporal overlap (articulatory phasing). The maximum simulated changes in segment duration that could be obtained with a phase-only model were small compared to the increases demonstrated by human speaker. Moreover, isolated changes in inter-articulatory timing (without other changes in the speed or distance of movement) resulted in apparent instabilities in the form of the F2 pattern. The bottom of Figure 2 displays the formant patterns that resulted from simultaneously changing the phasing and the speed of different articulators. Note the apparent increase in the homogeneity of the acoustic patterns across rates. These findings underscore the complexity of the problem of inferring between different levels of analysis.

Figure 2. The top panel displays the acoustic effects of speaking rate change simulated by only changing the phasing of articulatory movements with an articulatory synthesizer. Note the apparent instabilities in the resulting acoustics pattern. The bottom panel displays the apparently stabilizing influence of adding simulated changes in articulatory speed.
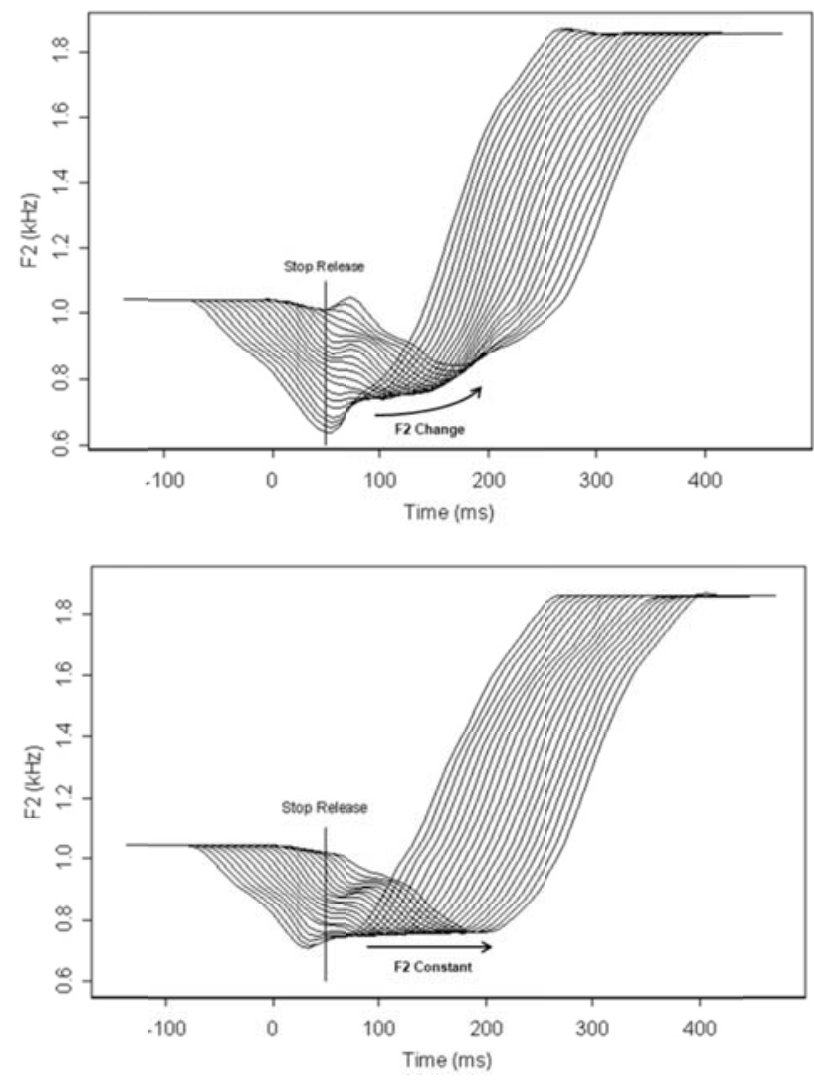

\section{Speaking Rate and Articulatory-Acoustic Relations}

The modeling results reported by Berry (2002) suggest that apparently distinct individual strategies defined kinematically (e.g., speed change versus phase change) may work synergistically to stabilize acoustic output. Thus, a speaker-general goal, such as the 
production of a stable acoustic output, may condition individual differences at the kinematic level of analysis, due to variation in articulatory-acoustic mapping between speakers. Mefferd and Green (2010) reported a unique attempt to study the relationship between articulatory and acoustic variability during speaking rate changes across three rates produced by 10 speakers. They computed spatiotemporal index values (Smith, Johnson, McGillem, \& Goffman, 2000) to describe variability in tongue sensor distances and acoustic (formant) distances between the vowels /i/ and /a/ across rate and clarity conditions. The authors reported a poor association between kinematic and acoustic variability and suggested that the relative acoustic stability could be the synergistic result of articulatory changes engaged to maximize acoustic stability. While such an interpretation is consistent with theoretical perspectives pertinent to articulatory-acoustic relations (c.f., Maeda, 1991; Stevens, 1989), a persistent challenge in appraising the adequacy of such an explanation derives from the wide diversity of phonetic content and speaking task. To bolster experimental control and create a manageable quantity of data to analyze, phonetic content and speaking task are often narrowly controlled in rate studies.

Tasko and McClean (2004) reported a unique appraisal of the interaction between the kinematic effects of rate change and speaking task. They studied tongue, lip, and jaw movements for 15 male talkers producing repetitions of a nonsense utterance and a sentence at three self-determined rates. Across subjects, movement duration was longest for slow, moderate for habitual, and shortest for fast rate replicates. Speed and distance decreased from habitual to slow, but neither variable followed a statistically significant trend in changing from habitual to fast rate. These effects were not equivalent across articulator. In particular, duration comparisons between habitual-slow and habitual-fast were non-significant for the tongue; distance comparisons were non-significant for the lower lip and mandible, and speed comparisons were non-significant for mandible. Most notably, duration and distance effects interacted with speech task. Duration was more affected in nonsense productions than in real sentences, whereas distance was more affected in sentences than nonsense productions. Overall, the authors demonstrated that speaking task and articulator interact with rate effects in complex ways. The potential effect of such interactions on experimental design and the generalizability of experimental results must be strongly considered by researchers and research consumers.

\section{Acoustic Effects of Speaking Rate Change}

Despite the preceding discussion, which suggests that acoustic effects of rate change may be small (relative to kinematic variability), studies of the acoustic effects of speaking rate change have identified a variety of speaker and context-related effects on formants (Agwuele, Sussman, \& Lindblom, 2008; Fourakis, 1991; Gay, 1968, 1978; Hertrich \& Ackermann, 1995; Lindblom, 1963; Pitermann, 2000; Rosen et al., 2011; Tjaden \& Weismer, 1998; van Son \& Pols, 1992; Weismer \& Berry, 2003). Taken together, these studies indicate a wide range of possible speaker-specific and context-related effects of speaking rate change. Evidence has been documented for changes in discrete-time formant measures, changes in formant transition slope, changes in the size of acoustic vowel space, and changes in the shape of formant patterns. The most commonly reported finding of discrete-time acoustic studies reflects "undershoot" of the formant target values with increasing rate (Lindblom, 1963).

Because of the relatively greater context sensitivity of F2 compared to F1 and F3 (Stevens \& House, 1963), effects on F2 have received the most attention. For example, Weismer and Berry (2003) reported rate-induced changes in both discrete-time acoustic parameters (e.g., F2 onset and F2 target values) and F2 trajectory shapes within and across 6 speakers producing a wide range of speaking rates. Speaker and (vowel) context-conditioned differences in the rate-induced effects on discrete-time parameters were pervasive. These findings may not be surprising given that context-related changes in formant shape may be impractically complex to describe mathematically (Broad \& Clermont, 1987). 


\section{Design and Analysis Issues}

The majority of studies of speaking rate variation have used designs that compare outcome measures between a few nominal rate conditions (e.g., slow, habitual, fast). A dependent measure of rate variation (e.g., segment duration) is often compared across conditions to confirm that subjects produced different responses between conditions. However, following such preliminary analysis, many studies neglect to treat rate as a continuous (dependent) variable, opting instead only to compare the effects on other dependent variables (e.g., speed, distance) across rate conditions. While such an approach may seem reasonable for bolstering scientific control and interpretability, it is unclear that any theoretical justification exists for treating speaking rate as a categorical variable. Moreover, it is well established that categorical treatment of a continuous variable can have several negative and potentially misleading statistical effects (c.f., MacCallum, Zhang, Preacher, \& Rucker, 2002; Royston, Altman, \& Sauerbrei, 2006; Taylor \& Yu, 2002).

This concern begs the question of whether speaking rate should be conceptualized as a continuous or categorical construct. This question pertains to speaking rate as a "speakergeneral" phenomenon and speaking rate variation within a particular speaker. While many speech scientists would agree that there are individual differences in speaker's preferred rate, we currently have a very limited understanding of what the normal range of rate variation is and how and why speakers differ. The limited research addressing this issue has focused on the dichotomy between "fast" and "slow" talkers (Crystal \& House, 1988) and involves only a relatively small literature. Categorical differentiation across speakers is a distinct concern from categorical differentiation of behaviors exhibited within speakers. Yet, in the context of the empirical study of speaking rate, it is likely that these two concerns interact in troubling ways.

Tsao and Weismer $(1997,2006)$ proposed that habitual rate differences between speakers may be influenced by individual differences in neuromuscular capabilities. Consistent with this hypothesis, they found that "slow" talkers produced maximum rates that approximated the habitual rates of "fast" talkers. This finding suggests a potential analytic problem for cross-subject pooling of rate data defined categorically and a potential speakergeneral confound in characterizing the effects of rate change.

The pooling problem may occur due to the possibility that categorically distinct behaviors may be wrongly treated as homogeneous. Assuming that a categorical rate elicitation task evokes ordinal changes in some quantitative index of speaking rate (within speaker), pooling across speakers (within categories) could result in data that differ (statistically) between categories - thus, apparently confirming that slow, habitual, and fast are "different" categories. Yet, the constituency of data within each category may represent very different locations along the global (speaker-general) continuum of speaking rate. As a consequence, comparisons of a dependent variable, such as articulatory speed or distance, across categories of rate may well be compromised by the erroneous presumption that the categories are constituted by comparable data.

The speaker-general confound may result from attempting to solve the pooling problem. For example, data could simply be categorized based on a quantitative measure of rate. By defining cut points along the rate continuum, categories could be defined to include data within a specified range of the quantitative measure of rate (much like a histogram). This approach might be justified by the natural occurrence of multiple modes along the continuum of a quantitative measure of rate. However, data presented by Weismer and Berry (2003) do not seem to support the assumption of a clearly multi-modal rate distribution. Moreover, even if such a natural justification for categorization existed, post-hoc categorization could still result in different individual speakers' contributing more or less data to each rate category. Specifically, the slow rate data would be populated primarily by tokens exhibited by the slowest speakers; the habitual category would be populated by a mixture of slow and fast talkers; and the fast rate data would be constituted primarily by data from the fast talkers. Thus, an analysis of the distinction between rate categories would be confounded by differences between 
speakers (with respect to preferred rates and ranges of rate change). Such a dilemma may be resolved only by improving upon our understanding of the normal range of variation in speaking rate, so that screening measures can be developed that will allow control for individual differences in habitual speaking rate.

\section{Conclusions}

The study of the articulatory effects of speaking rate change poses a host of challenging problems in speech science. Speech kinematic characterizations of speaking rate change have focused primarily on measures of movement speed, distance, and phase. Despite a substantial body of research, many studies report primarily idiosyncratic results. An improved capacity for the characterization of speaker-general effects of speaking rate may evolve through improved understanding of articulatory-acoustic relations and improvements in the adequacy of experimental design and data analysis methods.

\section{References}

Abbs, J. H. (1973). The influence of the gamma motor system on the jaw movements during speech: A theoretical framework and some preliminary observations. Journal of Speech and Hearing Research, 16, 175-200.

Adams, S. G., Weismer, G., \& Kent, R. D. (1993). Speaking rate and speech movement velocity profiles. Journal of Speech and Hearing Research 36, 41-54.

Agwuele, A., Sussman, H. M., \& Lindblom, B. (2008). The effect of speaking rate on consonant vowel coarticulation. Phonetica, 65, 194-209.

Benguerel, A., \& Cowan, H. A. (1974). Coarticulation of upper lip protrusion in French. Phonetica, 30, 4155.

Berry, J. (2002). Acoustic effects of speaking rate change in articulatory models. Journal of the Acoustical Society of America, 112, 2442(A).

Berry, J. (2003). Second formant effects of low dimensional models of speaking rate change in articulatory synthesis. Unpublished doctoral dissertation, University of Wisconsin-Madison.

Blanchet, P.G., \& Snyder, G.J. (2010). Speech rate treatments for individuals with dysarthria: A tutorial. Perceptual and Motor Skills, 110, 965-82.

Blomgren, M., \& Goberman, A. (2008). Revisiting speech rate and utterance length manipulations in stuttering speakers. Journal of Communication Disorders, 41,159-178.

Boyce, S. E., Krakow, R. A., Bell-Berti, F., \& Gelfer, C. E. (1990). Converging sources of evidence for dissecting articulatory movements into core gestures. Journal of Phonetics, 18, 173-188.

Broad, D. J., \& Clermont, F. (1987). A methodology for modeling vowel formant contours in CVC context. Journal of the Acoustical Society of America, 81, 155-165.

Byrd, D., \& Tan, C. C. (1996). Saying consonant clusters quickly. Journal of Phonetics, 24, $263-282$.

Crystal, T. H., \& House, A. S. (1988). A note on the variability of timing control. Journal of Speech and Hearing Research, 31, 497-502.

Davidow, J. H., Bothe, A. K., Richardson, J. D., \& Adreatta, R. D. (2010). Systematic studies of modified vocalization: Effects of speech rate and instatement style during metronome stimulation. Journal of Speech, Language and Hearing Research, 53, 1579-1594.

Engstrand, O. (1988). Articulatory correlates of stress and speaking rate in Swedish vcv utterances. Journal of the Acoustical Society of America, 83, 1863-1875.

Flege, J. E. (1988). Effects of speaking rate on tongue position and velocity of movement in vowel production. Journal of the Acoustical Society of America, 84, 901-916.

Fourakis, M. (1991). Tempo, stress, and vowel reduction in American English. Journal of the Acoustical Society of America, 90, 1816-1827.

Gay, T. (1968). Effect of speaking rate on diphthong formant movements. Journal of the Acoustical Society of America, 44, 1570-1573. 
Gay, T. (1978). Effect of speaking rate on vowel formant movements. Journal of the Acoustical Society of America, 63, 223-230.

Gay, T. (1981). Mechanisms in the control of speech rate. Phonetica, 38, 148-151.

Gay, T., \& Hirose, H. (1973). Effect of speaking rate on labial consonant production. Phonetica, 27, 44-56.

Gay, T., Ushijima, T., Hirose, H., \& Cooper, F. S. (1974). Effect of speaking rate on labial consonant-vowel articulation. Journal of Phonetics, 2, 47-63.

Guenther, F. H. (1995). Speech sound acquisition, coarticulation, and rate effects in a neural network model of speech production. Psychological Review, 102, 594-621.

Goozee, J., Lapointe, L., \& Murdoch, B. (2003). Effects of speaking rate on EMA-derived lingual kinematics: a preliminary investigation. Clinical Linguistics and Phonetics, 17, 375-381.

Goozee, J., Stephenson, D., Murdoch, B., Darnell, R., \& Lapointe, L. (2005). Lingual kinematic strategies used to increase speech rate: comparison between younger and older adults. Clinical Linguistics and Phonetics, 19, 319-334.

Hertrich, I., \& Ackermann, H. (1995). Coarticulation in slow speech: Durational and spectral analysis. Language and Speech, 38, 159-187.

Hertrich, I., \& Ackermann, H. (2000). Lip-jaw and tongue-jaw coordination during rate-controlled syllable repetitions. Journal of the Acoustical Society of America, 107, 2236-2247.

Kelso, J. A. S., Vatikiotis-Bateson, E., Saltzman, E. L., \& Kay, B. (1985). A qualitative dynamic analysis of reiterant speech production: phase portraits, kinematics, and dynamic modeling. Journal of the Acoustical Society of America, 77, 266-280.

Kent, R. D., \& Moll, K. L. (1972). Cinefluorographic analyses of selected lingual consonants. Journal of Speech and Hearing Research, 15, 453-473.

Kuehn, D. P., \& Moll, K. L. (1976). A cinderadiographic study of vc and cv articulatory velocities. Journal of Phonetics, 4, 303-320.

Lindblom, B. (1963). Spectrographic study of vowel reduction. Journal of the Acoustical Society of America, 35, 1773-1781.

Lindblom, B. E. F., \& Studdert-Kennedy, M. (1967). On the role of formant transitions in vowel recognition. Journal of the Acoustical Society of America, 42, 830-843.

Logan, K. J., Roberts, R. R., Pretto, A. P., \& Morey, M. J. (2002). Speaking slowly: Effects of four selfguided training approaches on adults' speech rate and naturalness. American Journal of Speech-Language Pathology, 11, 163-174.

Maeda, S. (1991). On articulatory and acoustic variabilities. Journal of Phonetics, 19, 321-331.

MacCallum, R.C., Zhang, S., Preacher, K.J., \& Rucker, D.D. (2002). On the practice of dichotomization of quantitative variables. Psychological Methods, 7, 19-40.

McClean (2000). Patterns of orofacial movement velocity across variations in speech rate. Journal of Speech, Language and Hearing Research, 43, 205-216.

McClean, M., \& Tasko, S. (2003). Association of orofacial muscle activity and movement during changes in speech rate and intensity. Journal of Speech, Language, and Hearing Research, 46, 1387-1400.

Mefferd, A. S., \& Green, J. R. (2010). Articulatory-to-acoustic relations in response to speaking rate and loudness manipulations. Journal of Speech, Language, and Hearing Research, 53, 1206-1219.

Munhall, K. G., Ostry, D. J., \& Parush, A. (1985). Characteristics of velocity profiles of speech movements. Journal of Experimental Psychology: Human Perception and Performance, 11, 457-474.

Nelson, W. L. (1983). Physical principles for economies of skilled movement. Biological Cybernetics, 46 , 135-147.

Nishio, M., \& Niimi, S. (2001). Speaking rate and its components in dysarthric speakers. Clinical Linguistics and Phonetics, 15, 309-317.

Nishio, M., \& Niimi, S. (2006). Comparison of speaking rate, articulation rate and alternating motion rate in dysarthric speakers. Folia Phoniatrica et Logopaedica, 58, 114-131.

Ostry, D. J., Cooke, J. D., \& Munhall, K. G. (1987). Velocity curves of human arm and speech movements. Experimental Brain Research, 68, 37-46. 
Ostry, D. J., \& Munhall, K. G. (1985). Control of rate and duration of speech movements. Journal of the Acoustical Society of America, 77, 640-648.

Perkell, J. S., Zandipour, M., Matthies, M. L., \& Lane, H. (2002). Economy of effort in different speaking conditions. I. A preliminary study of intersubject differences and modeling issues. Journal of the Acoustical Society of America, 112, 1627-1641.

Pitermann, M. (2000). Effect of speaking rate and contrastive stress on formant dynamics and vowel perception. Journal of the Acoustical Society of America, 107, 3425-3437.

Plamondon, R. (1995). A kinematic theory of rapid human movements: Part II movement and time control. Biological Cybernetics, 72, 309-320.

Rosen, K. M., Folker, J. E., Murdoch, B. E., Vogel, A. P., Cahill, L. M., Delatycki, M. B., \& Corben, L. A. (2011). Measures of spectral change and their application to habitual, slow, and clear speaking modes. International Journal of Speech-Language Pathology, 13, 165-173.

Royston, P., Altman, D. G., \& Sauerbrei, W. (2006). Dichotomizing continuous predictors in multiple regression: a bad idea. Statistics in Medicine, 25, 127-141.

Saltzman, E., \& Byrd, D. (2000). Task dynamics of gestural timing: Phase windows and multifrequency rhythms. Human Movement Science, 19, 499-526.

Saltzman, E. L., \& Munhall, K. G. (1989). A dynamical approach to gestural patterning in speech production. Ecological Psychology, 1, 333-383.

Shaiman, S. (2001). Kinematics of compensatory vowel shortening: The effect of speaking rate and coda composition on intra- and inter-articulatory timing. Journal of Phonetics, 29, 89-107.

Shaiman, S. (2002). Articulatory control of vowel length for contiguous jaw cycles: The effects of speaking rate and phonetic context. Journal of Speech, Language, and Hearing Research, 45, 663-675.

Shaiman, S., Adams, S. G., \& Kimelman, M. D. Z. (1995). Timing relationships of the upper lip and jaw across changes in speaking rate. Journal of Phonetics, 23, 119-128.

Shaiman, S., Adams, S. G., \& Kimelman, M. D. Z. (1997). Velocity profiles of lip protrusion across changes in speaking rate. Journal of Speech and Hearing Research, 40, 144-158.

Smith, A., Johnson, M., McGillem, C., \& Goffman, L. (2000). On the assessment of stability and patterning of speech movements. Journal of Speech, Language, and Hearing Research, 43, $277-286$.

Stetson, R. H. (1951). Motor phonetics. Amsterdam, Holland: North-Holland.

Stevens, K. N. (1989). On the quantal nature of speech. Journal of Phonetics, 17, 3-45.

Stevens, K. N., \& House, A. S. (1963). Perturbation of vowel articulations by consonantal context: An acoustical study. Journal of Speech \& Hearing Research, 6, 111-128.

Stone, M. (1997). Laboratory techniques for investigating speech articulation. In W. J. Hardcastle \& J. Laver (Eds.), The handbook of phonetic sciences (pp. 11-32). Blackwell, UK: Oxford.

Tasko, S., \& Westbury, J. R. (2002). Defining and measuring speech movements. Journal of Speech, Language, and Hearing Research, 45, 127-142.

Tasko, S., \& McClean, M. (2004). Variations in articulatory movement with changes in speech task. Journal of Speech, Language and Hearing Research, 47, 85-100.

Taylor, J. M. G., \& Yu, M. (2002). Bias and efficiency loss due to categorizing an explanatory variable. Journal of Multivariate Analysis, 83, 248-263.

Tjaden, K., \& Weismer, G. (1998). Speaking-rate-induced variability in F2 trajectories. Journal of Speech, Language and Hearing Research, 41, 976-989.

Tsao, Y. C., \& Weismer, G. (1997). Interspeaker variation in habitual speaking rate: evidence for a neuromuscular component. Journal of Speech, Language, and Hearing Research, 40, 858-866.

Tsao, Y. C., \& Weismer, G. (2006). Interspeaker variation in habitual speaking rate: Additional evidence. Journal of Speech, Language, and Hearing Research, 49, 1156-1164.

van Son, R. J. J. H., \& Pols, L. C. W. (1992). Formant movements of Dutch vowels in a text, read at normal and fast rate. Journal of the Acoustical Society of America, 92, 121-127.

Viviani, P., \& Cenzato, M. (1985). Segmentation and coupling in complex movements. Journal of Experimenal Psychology: Human Perception and Performance, 11, 828-845. 
Weismer, G., Tjaden, K., \& Kent, R. (1995). Can articulatory behavior in motor speech disorders be accounted for by theories of normal speech production. Journal of Phonetics, 23, 149-164.

Weismer, G., \& Berry, J. (2003). Effects of speaking rate on second formant trajectories of selected vocalic nuclei. Journal of the Acoustical Society of America, 113, 1-17.

Yorkston, K., Hakel, M., Beukelman, D., \& Fager, S. (2007). ANCDS bulletin board. Evidence for effectiveness of treatment of loudness, rate, or prosody in dysarthria: a systematic review. Journal of Medical Speech-Language Pathology, 15, xi-xxxvi. 